International Journal of Pure and Applied Mathematics

Volume 94 No. 5 2014, 697-700

ISSN: 1311-8080 (printed version); ISSN: 1314-3395 (on-line version)

url: http://www.ijpam.eu

doi: http://dx.doi.org/10.12732/ijpam.v94i5.7

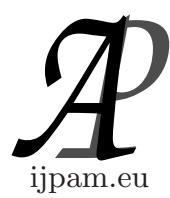

\title{
BINOMIAL APPROXIMATION FOR RANDOM SUMS OF BERNOULLI RANDOM VARIABLES
}

\author{
K. Teerapabolarn \\ Department of Mathematics \\ Faculty of Science \\ Burapha University \\ Chonburi, 20131, THAILAND
}

\begin{abstract}
In this paper, we give a bound for the total variation distance between the distribution of random sums of independent Bernoulli random variables and a binomial distribution. Two examples have been given to illustrate the result obtained.
\end{abstract}

AMS Subject Classification: 62E17, 60F05, 60G05

Key Words: Bernoulli random variable, binomial approximation, random sums, total variation distance

\section{Introduction}

Let $X_{1}, X_{2}, \ldots$ be a sequence of independent Bernoulli random variables, each with probability $p_{i}=1-q_{i}=P\left(X_{i}=1\right)=1-P\left(X_{i}=0\right)$. Let $S_{N}=\sum_{i=1}^{N} X_{i}$, where $N$ is a non-negative integer-valued random variable and independent of the $X_{i}$ 's. The random summands is usually called random sums. Let $B_{n, p}$ be a binomial random variable with parameters $n$ and $p$. For $N=n \in \mathbb{N}$ is fixed, Ehm [2] gave a bound for approximating the distribution of $S_{n}$ by a binomial distribution in the form of

Received: March 26, 2014

(c) 2014 Academic Publications, Ltd. url: www.acadpubl.eu 


$$
d_{T V}\left(S_{n}, B_{n, p}\right) \leq \frac{1-p^{n+1}-q^{n+1}}{(n+1) p q} \sum_{i=1}^{n}\left(p_{i}-p\right)^{2},
$$

where $d_{T V}\left(S_{n}, B_{n, p}\right)=\sup _{A \subseteq\{0,1, \ldots, n\}}\left|P\left(S_{n} \in A\right)-P\left(B_{n, p} \in A\right)\right|$ is the total variation distance between the distribution of $S_{n}$ and the binomial distribution with parameters $n$ and $p=1-q=\frac{1}{n} \sum_{i=1}^{n} p_{i}$. Let $\widehat{n}=E(N)$ and $\widehat{p}=1-\widehat{q}=\frac{\lambda}{\hat{n}}$, where $\widehat{n} \in \mathbb{N}$ and $\lambda=E\left(\lambda_{N}\right)=E\left(\sum_{i=1}^{N} p_{i}\right)$. In this study, we are interested to determine a bound for $d_{T V}\left(S_{N}, B_{\widehat{n}, \widehat{p}}\right)$, which is in Section 2. In Section 3, two examples have been given to illustrate the desired result, and the conclusion of this study is presented in the last section.

\section{Result}

We give a bound for the total variation distance between the distributions of $S_{N}$ and $B_{\widehat{n}, \widehat{p}}$ as follows.

Theorem 2.1. For $\lambda_{N}=\sum_{i=1}^{N} p_{i}, \lambda=E\left(\lambda_{N}\right)$ and $\widehat{n} \in \mathbb{N}$, then

$$
d_{T V}\left(S_{N}, B_{\widehat{n}, \widehat{p}}\right) \leq\left(1-e^{-\lambda}\right)\left\{\frac{\operatorname{Var}\left(\lambda_{N}\right)}{\lambda}+\frac{\lambda}{\widehat{n}}\right\}+E\left(\frac{1-e^{-\lambda_{N}}}{\lambda_{N}} \sum_{i=1}^{N} p_{i}^{2}\right)
$$

Proof. Let $U_{\lambda}$ be the Poisson random variable with mean $\lambda$. It follows the fact that

$$
\begin{aligned}
d_{T V}\left(S_{N}, B_{\widehat{n}, \widehat{p}}\right) & \leq d_{T V}\left(S_{N}, U_{\lambda}\right)+d_{T V}\left(U_{\lambda}, B_{\widehat{n}, \widehat{p}}\right) \\
& \leq d_{T V}\left(S_{N}, U_{\lambda_{N}}\right)+d_{T V}\left(U_{\lambda_{N}}, U_{\lambda}\right)+d_{T V}\left(U_{\lambda}, B_{\widehat{n}, \widehat{p}}\right)
\end{aligned}
$$

Following [3], we have

$$
d_{T V}\left(S_{N}, U_{\lambda_{N}}\right) \leq E\left(\frac{1-e^{-\lambda_{N}}}{\lambda_{N}} \sum_{i=1}^{N} p_{i}^{2}\right)
$$

Applying Theorem 1.C and using inequality (1.23) in [1], we also obtain

$$
d_{T V}\left(U_{\lambda_{N}}, U_{\lambda}\right) \leq\left(1-e^{-\lambda}\right) \frac{\operatorname{Var}\left(\lambda_{N}\right)}{\lambda}
$$

and

$$
d_{T V}\left(U_{\lambda}, B_{\widehat{n}, \widehat{p}}\right) \leq\left(1-e^{-\lambda}\right) \widehat{p}=\left(1-e^{-\lambda}\right) \frac{\lambda}{\widehat{n}}
$$


respectively. Hence, the inequality (2.1) is obtained by taking the bounds in (2.3), (2.4) and (2.5) into (2.2).

If $X_{i}$ 's are identically distributed, then the following corollary is an immediately consequence of the Theorem 2.1

Corollary 2.1. For $\widehat{n} \in \mathbb{N}$, if $p_{1}=p_{2}=\cdots=p$, then we have the following:

$$
d_{T V}\left(S_{N}, B_{\widehat{n}, p}\right) \leq\left(1-e^{-\widehat{n} p}\right)\left\{\frac{\operatorname{Var}(N)}{\widehat{n}}+1\right\} p+E\left(1-e^{-N p}\right) p .
$$

\section{Examples}

This section, two examples are given to illustrate the result in the case of $X_{i}$ 's are identically distributed.

Example 3.1. For $n(n \in \mathbb{N})$ is fixed, let $N$ be a positive integer-valued random variable with probability function

$$
P(N=k)= \begin{cases}\frac{1}{2} & , k=2 n \\ \frac{1}{2} & , k=4 n \\ 0 & , \text { otherwise }\end{cases}
$$

Therefore $\widehat{n}=3 n$ and $\operatorname{Var}(N)=n^{2}$. Let $p_{1}=p_{2}=\cdots=p$, then we have

$$
d_{T V}\left(S_{N}, B_{3 n, p}\right) \leq\left(1-e^{-3 n p}\right) \frac{(n+3) p}{3}+\left(1-\frac{e^{-2 n p}+e^{-4 n p}}{2}\right) p .
$$

Example 3.2. Let $N$ be a positive integer-valued random variable with probability function

$$
P(N=n)=\frac{1}{20}, n=1,2, \ldots, 21,
$$

then we have $\widehat{n}=11$ and $\operatorname{Var}(N)=\frac{891}{20}$. If $p_{1}=p_{2}=\cdots=p$, then we obtain

$$
d_{T V}\left(S_{N}, B_{11, p}\right) \leq\left\{\left(1-e^{-11 p}\right) \frac{101}{20}+1\right\} p .
$$




\section{Conclusion}

In this study, a bound for the total variation distance between the distribution of random sums of independent Bernoulli random variables and an appropriate binomial distribution was obtained. It is indicated that the binomial with parameters $\widehat{n}$ and $\widehat{p}$ can be used as an estimate of the distribution of random sums of independent Bernoulli random variables when $\widehat{p}$ is small.

\section{References}

[1] A.D. Barbour, L. Holst and S. Janson, Poisson Approximation, Oxford Studies in probability 2, Clarendon Press, Oxford, 1992.

[2] W. Ehm, Binomial approximation to the Poisson binomial distribution, Statist. Probab. Lett., 11 (1991), 7-16.

[3] N. Yannaros, Poisson approximation for random sums of Bernoulli random variables, Statist. Probab. Lett., 11 (1991), 161-165. 